médecine/sciences $85 ; 1: 36-40$

\title{
La leucémie à tricholeucocytes: une indication privilégiée de l'interféron alpha
}

La leucémie à tricholeucocytes, récemment individualisée, représente environ $2 \%$ de l'ensemble des leucémies. L'efficacité régulière de l'interféron dans son traitement, rapportée ici dans la première communication originale française sur le sujet, ouvre de nouvelles perspectives dans l'usage de cette interleukine.

Georges Flandrin François Sigaux Sylvie Castaigne Christian Billard Laurent Degos Ernesto Falcoff

\section{REFERENCES}

I. Bouroncle B. A., Wiseman B. K., Doan C. A. Leukemic reticulo-endotheliosis. Blood 1958; 13: 609-30.

2. Flandrin G., Sigaux F., Sébahoun G., Bouffette P. Hairy cell leukemia. Clinical presentation and follow-up of 211 patients. Semin Oncol 1984; 11 : $333-46$.

3. Golomb M., 'Hadad J. Infections complications in 127 patients with hairy cell leukemia. $\mathrm{Am}$ J Hematol 1984; $16: 393-401$.

\section{ADRESSES}

G. Flandrin, F. Sigaux, S. Castaigne, L. Degos Hôpital Saint-Louis. 2, place du DocteurFournier. 75475 Paris Cedex 10.

C. Billard, E. Falcoff : Institut Curie. 26, rue a leucémie à tricholeucocytes (hairy cell leukemia) est une affection tumorale du tissu hématopoḯtique d'individualisation beaucoup plus récente que les autres variétés d'hémopathies malignes. Ce retard a été dû à la difficulté d'obtenir facilement les cellules anormales par la technique habituelle de ponctionaspiration, du fait de l'existence d'une fibrose de la moelle osseuse. C'est l'introduction de la biopsie médullaire en pratique courante dans les années 1960 , permettant l'étude d'un fragment de tissu médullaire avec les cellules en place, qui a facilité l'identification de cette variété nouvelle de leucémie [I]. Les premiers travaux se sont surtout attachés à affiner les critères diagnostiques et à établir les premières descriptions cliniques de cette maladie, d'abord jugée comme exceptionnelle. Depuis une dizaine d'années, c'est le débat sur la nature exacte de la cellule tumorale en cause qui a surtout suscité travaux et controverses ( $f i g$. I ). Enfin, dans le même temps, avec l'apparition de plus grandes séries cliniques, l'évaluation des critères de gravité (pronostic) et l'établissement des indications thérapeutiques ont fait l'objet d'une recherche clinique. Jusqu'à ces derniers temps, seule la splénectomie était d'indication non contestée. La démonstration toute récente d'une efficacité régulière de l'alpha-interféron est venue boule- verser les termes du problème clinique posé par cette affection et justifie le renouveau d'intérêt porté à la leucémie à tricholeucocytes.

\section{Description hématologique}

La fréquence des symptômes de la description qui va suivre est basée sur l'expérience de l'hôpital SaintLouis, portant en 1983 sur l'étude de 2 I I cas, ces constatations étant convergentes avec celles des autres séries de la littérature [2].

La fréquence de l'affection est estimée à environ $2 \%$ de l'ensemble des leucémies. La prédominance masculine est importante ( 3,6 pour I). L'âge moyen de découverte est comparable dans les deux sexes, autour de 52 ans, l'affection ne s'observant que chez l'adulte (âges extrêmes de 23 à 89 ans, plus de la moitié des malades entre 40 et 60 ans). Le plus souvent, les signes révélateurs sont les conséquences de l'anémie - faiblesse, fatigabilité ( $27 \%$ des cas), auxquelles s'associent des manifestations hémorragiques discrètes, témoins de la thrombopénie elle-même rarement révélatrice $(4 \%)$ et plus souvent encore, des manifestations infectieuses $(29 \%)$, notamment respiratoires, témoins de la neutropénie.

La splénọmégalie est la constatation d'examen la plus fréquente $(72 \%$ des cas ont une rate palpable, dans 
I $8 \%$ des cas elle est. très importante, dépassant de plus de $10 \mathrm{~cm}$ le rebord costal). Une hépatomégalie modérée a été notée dans $20 \%$ des cas. Toutes les aires ganglionnaires superficielles sont habituellement libres. Les seules manifestations cutanées sont hémorragiques (purpura et ecchymoses) ou infectieuses, pouvant avoir un aspect nécrotique, mais sans infiltration spécifique. Une pancytopénie est fréquente dès le premier examen du sang $(59 \%$ des cas) avec pour limites : hémoglobine $(\mathrm{Hb})$ I2 $\mathrm{g} / \mathrm{dl}$, plaquettes $(\mathrm{Pl})$ I50. 10 $9 / 1$, polynucléaires neutrophiles (Pn) 2. $10^{9} / 1$. La neutropénie surtout est le fait majeur par sa constance et sa netteté. Une mono- cytopénie, difficile à évaluer précisément, est également très fréquente. Si la plupart des malades sont leucopéniques, quelques-uns ( $10 \%$ ) ont plus de $10.109 / 1$ globules blancs. Cette hyperleucocytose est due au passage sanguin de tricholeucocytes. L'anémie est fréquente, souvent modérée (taux de l'hémoglobine inférieur à $12 \mathrm{~g} / \mathrm{dl}$ dans $70 \%$ des cas ou à $8 \mathrm{~g} / \mathrm{dl}$ dans I6\% des cas). S'il s'agit souvent d'une anémie vraie par trouble de production médullaire (taux bas des réticulocytes, diminution de l'incorporation du fer), la surestimation de cette anémie est habituelle dans les formes splénomégaliques, du fait de l'hémodilution. Cette hémodilution

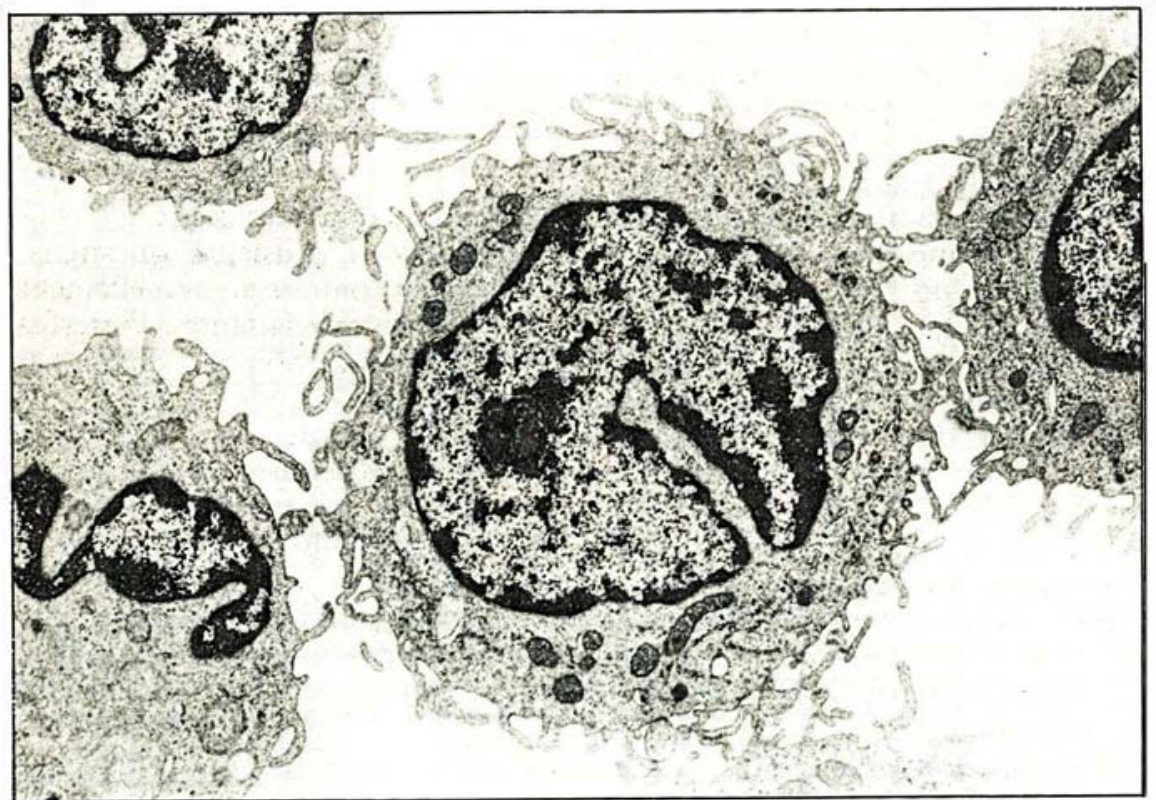

Figure 1. Les tricholeucocytes (Cliché du Dr. M. T. Daniel. Aspect ultrastructural. Agrandissement : $\times 6750)$. Sur frottis coloré, cette cellule possède un noyau arrondi ou ovalaire, une chromatine claire, un cytoplasme étendu faiblement basophile, à limites hérissées de fins prolongements. Cet aspect "chevelu", qui a valu son nom aux tricholeucocytes, est surtout évident sur les cellules vivantes observées en contraste de phase ou sur les images ultrastructurales. Ces cellules possèdent une activité modérée estérase non spécifique et une activité phosphatase acide particulière, résistant $a^{\star} l$ 'acide tartrique. La morphologie ambiguë de ces cellules a fait discuter leur origine, d'autant plus qu'elles semblent posséder certaines propriétés des cellules lymphoides $B$ [8] et des monocytes. Outre des propriétés phagocytaires, une capacité d'adhésion au verre, l'absence de $H L A-O Q$, elles ont une activité enzymatique du type peroxydase les différenciant des autres cellules lymphoïdes. Ces cellules possèdent souvent des immunoglobulines de membrane, un récepteur pour le fragment $F c$ des $\operatorname{Ig} G$ et des IgM (parfois un récepteur pour le complément) et des antigènes de la classe II d'histocompatibilité (HLA DR). Ces trois derniers marqueurs sont cependant communs aux monocytes et aux lymphocytes $B$. Des études in vitro de synthèse d'Ig par les tricholeucocytes sont compatibles avec la nature $B$ des cellules. La démonstration d'un réarrangement des gènes des immunoglobulines confirme définitivement cette hypothèse. Un récepteur pour l'interleukine 2, habituellement présent dans les cellules $T$ activées et certaines cellules $B$ activées, a également été retrouvé [9].

$\mathrm{m} / \mathrm{s}$ mars 85 peut être estimée par la mesure du volume plasmatique. Elle est en excellente corrélation avec la taille de la rate. Une thrombopénie de degré variable est habituellement constatée $(64 \%$ des cas ont moins de $100.10 \% / 1$ plaquettes et $30 \%$, moins de $\left.50.10^{9} \mid 1\right)$.

La ponction de mœlle avec aspiration ne ramène, en général, aucun grumeau et les étalements sont habituellement pauvres en cellules. La biopsie médullaire montre habituellement une mœlle très riche en cellules. Il existe quasi constamment une fibrose réticulinique. La fibrose est l'explication principale des échecs de l'aspiration médullaire. On constate une raréfaction plus ou moins importante des lignées myéloïdes normales.

\section{Évolution}

En dehors de leur asthénie, la plupart des malades ont un état général assez bien conservé au moment du diagnostic, mais également pendant la plus grande période de leur évolution clinique. Les épisodes infectieux représentent les complications cliniques les plus fréquentes, les plus graves et le plus souvent responsables des décès.

Dans une série clinique récente de I27 patients [3] Golomb et ses collaborateurs trouvent que $68,5 \%$ des patients ont eu des accidents infectieux au cours de leur évolution. Les patients sujets à ces accidents infectieux ont une durée de vie plus courte que les patients exempts d'infections $(49 \%$ contre $92 \%$ vivants à 4 ans). Les deux types d'infections les plus communs sont les septicémies (30 patients) et les pneumopathies (27 patients). Parmi les septicémies les plus fréquentes, il faut signaler E. Coli, $P$. Aeruginosa et $S$. Auréus. En outre, le risque de survenue d'infection à mycobactéries atypiques et d'une tuberculose est à considérer. La médiane actuarielle de survie dans notre série de 2 I I malades est de 53 mois. Quatre malades sont actuellement vivants, plus de 200 mois après le début de leur maladie. Au moment du diagnostic, une neutropénie $<0,5 \cdot 10^{9} / 1$ (médiane de survie 32 mois) et une thrombopé- 
nie $<50.10^{9} / 1$ (médiane de survie 29 mois) ont une importance pronostique péjorative. Le degré de l'anémie, l'hyperleucocytose et la taille de la rate ne semblent pas avoir d'incidence sur le pronostic. Un taux bas d'incorporation du fe médullaire, apprécié par étude isotopique, a une influence pronosti. que péjorative.

Le pronostic semble également dépendre du degré de l'infiltration ostéo-médullaire, indépendamment des autres facteurs [4].

Parmi les traitements, seule la splénectomie a montré une efficacité régulière. Dans les mois qui succèdent à la splénectomie, on observe dans la majorité des cas une correction des cytopénies périphériques, le degré d'amélioration n'étant pas lié à la taille de la rate. Les patients splénectomisés ont une survie significativement plus longue que celle des patients non splénectomisés ( fig. 2); ce résultat est d'autant plus intéressant que dans notre série, il n'existe pas de différence significative entre les deux groupes de patients pour les paramètres pronostiques principaux (chiffre des Pn et des $\mathrm{Pl}$ ). L'effet de la splénectomie sur la survie des patients ne devient significatif que pour les patients ayant une rate volumineuse (plus de $4 \mathrm{~cm}$ de débord sous le gril costal). Après splénectomie, l'absence de correction de l'anémie avec maintien des besoins transfusionnels, comme la persistance d'une neutropénie, sont des facteurs de mauvais pronostic. Les tentatives thérapeutiques autres que la splénectomie n'ont pas donné de résultats convainquants. Les faibles doses de chlorambucil permettent d'obtenir des corrections de l'anémie et de la thrombopénie mais sans effet notable sur la neutropénie. Les alcaloïdes de la pervenche, le phosphore-32, les androgènes sont très peu efficaces. La corticothérapie a parfois un effet temporaire mais semble majorer les risques infectieux. Les polychimiothérapies peuvent être proposées dans les formes les plus sévères mais si elles obtiennent parfois des rémissions complètes, elles sont grevées d'une très lourde mortalité $[5,6]$.

La première publication faisant état de l'efficacité de l'interféron alpha comme traitement de la leucémie à tricholeucocytes est celle de J. R. Quesada et collaborateurs du groupe de J. U. Gutterman [7]. Ces auteurs, en I984, faisaient état de trois rémissions complètes et de quatre rémissions partielles obtenues chez sept patients traités pendant quelques mois par doses faibles d'interféron alpha leucocytaire partiellement purifié. Ces résultats étaient remarquables par plusieurs points; c'était la première fois qu'on obtenait dans cette maladie avec une thérapeutique peu agressive des rémissions complètes vérifiées histologiquement; en outre les doses employées étaient plutôt faibles (3. $\left.10^{6} \mathrm{U} / \mathrm{j}\right)$ et n'étaient responsables que de peu d'effets secondaires.

\section{Traitement par l'interféron alpha}

Il restait à confirmer ces résultats et à répondre à plusieurs questions, dont deux ont reçu actuellement une réponse satisfaisante : l'interféron alpha recombinant donne-t-il des résultats analogues? Le traitement est-il efficace dans les formes très sévères (pancytopénies prof ondes non corrigées par la splénectomie et infections répétées)? L'effet de l'interféron alpha est-il stable à long terme? Permet-il d'allonger la survie des patients et, question liée, quelle est la durée optimale du traitement? Enfin quels sont les mécanismes d'action du traitement? A la suite de la publication de Quesada plusieurs équipes, dont la nôtre, mettaient en place des protocoles thérapeutiques utilisant l'interféron alpha cloné à faible dose; l'originalité de notre étude tenant au traitement selon des modalités voisines de trois groupes de patients par trois interférons alpha différents : deux interférons clonés (Hoffmann La Roche et Schering Corporation) et un interféron alpha leucocytaire (fourni généreusement par R. Cantell : Croix Rouge Finlandaise) identique à celui utilisé par Quesada et coll. Actuellement, 30 patients ont été inclus dans cette étude (dont 27 traités par interférons clonés). Un délai suffisant entre le début du traitement par interféron et un traitement antérieur 
(au moins 6 mois par une splénectomie et I mois par une chimiothérapie anti mitotique) a été respecté. Dans tous les cas, un effet thérapeutique a été observé. Bien que l'évolution sous traitement soit relativement variable d'un cas à un autre, on peut tenter de la schématiser de la façon suivante ( $\mathrm{fig} .3$ et 4 ). $\mathrm{Au}$ cours du premier mois, s'observe dans le sang une diminution du nombre des tricholeucocytes circulants, ainsi qu'une diminution du nombre absolu des polynucléaires neutrophiles, diminution transitoire mais parfois importante. Parallèlement chez des patients splénomégaliques, la taille de la rate diminue et la splénomégalie disparaît dans les deux premiers mois. La correction des cytopénies (anémie, neutropénie, monocytopénie et thrombopénie) ne s'observe généralement qu'après le deuxième mois. La thrombopénie est souvent la première des cytopénies à régresser, la correction de la monocytopénie, de la neutropénie et de l'anémie était en règle plus tardive. Au niveau médullaire, s'observe sur les biopsies faites après 4 mois de traitement, une diminution nette de l'infiltration par les tricholeucocytes et une augmentation du volume occupé par les lignées myélö̈des. A cette date, la majorité des patients
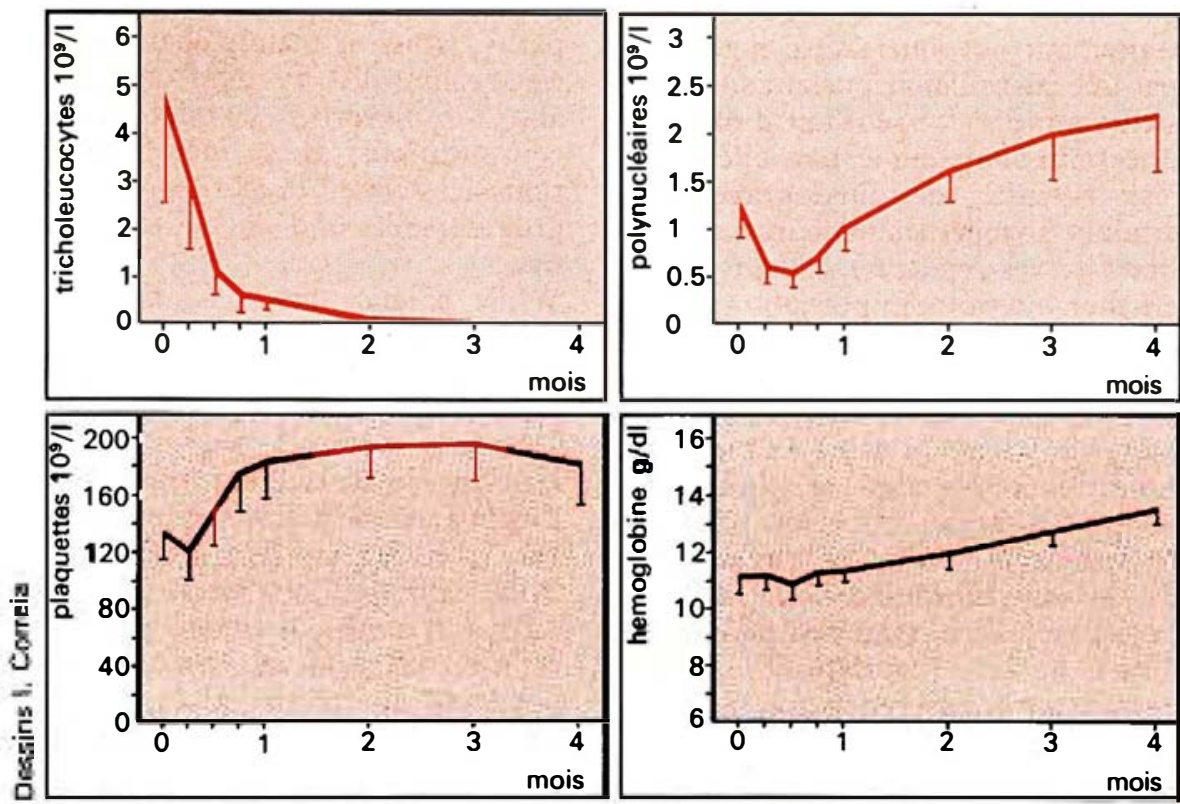

Figure 3. Evolution hématologique moyenne au cours du traitement pour l'ensemble des patients de l'étude. ( $\perp$ représente l'erreur standard de la moyenne).

$\mathrm{m} / \mathrm{s}$ mars 85

Figure 2. Courbe actuarielle de survie (méthode de Kaplan Meier) pour les patients splénectomisés ( 85 patients médiane non atteinte à 200 mois) et non splénectomisés (I26 patients - médiane 37 mois). Les courbes different significativement $(p<0$, or test du Log-rank).
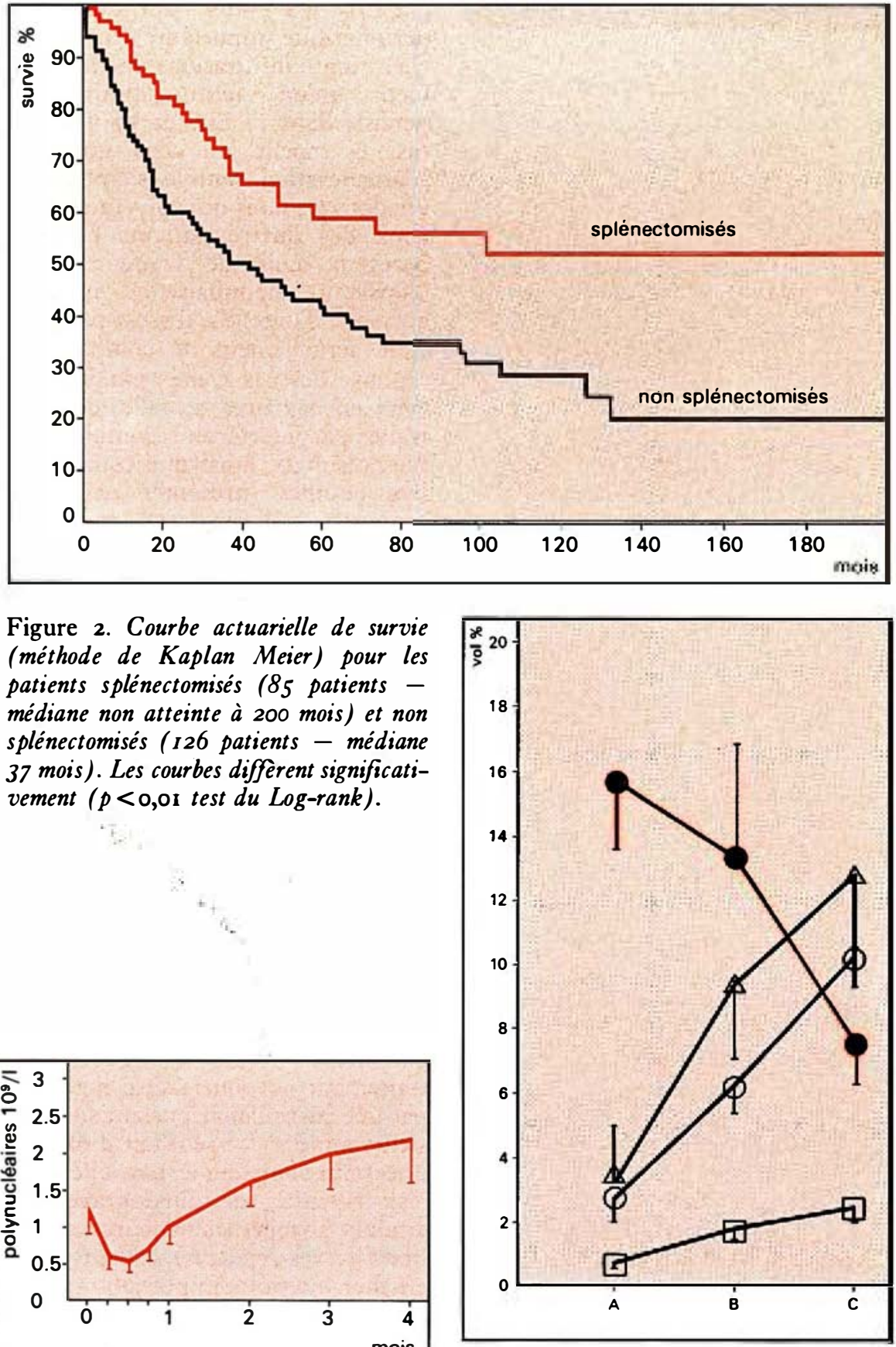

Figure 4. Evolution pour l'ensemble des patients de l'étude des volumes relatifs moyens occupés par ( $\square$ ) les mégacaryocytes, $(O)$ les erythroblastes, $(\triangle)$ les cellules granuleuses et (O) les tricholeucocytes dans les biopsies médullaires pratiquées (A) avant traitement, (B) après 2 mois de traitement et $(C)$ après 4 mois de traitement. Les volumes exprimés en pourcentages sont évalués à partir des surfaces occupées sur les coupes histologiques en utilisant une méthode stéréologique de comptage de points (I 000 points environ pour chaque biopsie). 
(12/17) n'a plus de cytopénie périphérique importante; en revanche, une infiltration médullaire, bien qu'en nette diminution, persiste dans i I cas sur I 7. Dans 3 cas, la moelle est déjà normale. L'amélioration semile se poursuivre dans les mois qui suivent et chez deux des quatre patients étudiés, après 6 mois de traitement, la biopsie médullaire apparaît normale. Toutefois, il n'est pas possible actuellement de comparer la réponse des cas traités par interféron leucocytaire, à celle des cas traités par interféron recombinant. Nos résultats, ainsi que ceux d'autres équipes (présentés dans des rapports préliminaires), montrent donc que l'interféron alpha permet d'obtenir régulièrement un effet thérapeutique dans la leucémie à tricholeucocytes. Il n'est cependant pas évident qu'une rémission médullaire complète puisse être obtenue dans chaque cas. Cette constatation n'enlève cependant pas forcément son intérêt à cette méthode thérapeutique. La cinétique très lente du tricholeucocyte devrait permettre d'éviter une repopulation trop rapide de la molle par les cellules malignes. Par ailleurs, la question essentielle est en fait celle de la stabilité de la correction des cytopénies périphériques. Il faut souligner que pour la majorité des patients, le bénéfice à long terme du traitement ne pourra être jugé que par une surveillance portant sur plusieurs années. Le seul fait d'obtenir une rémission même partielle chez les patients les plus gravement atteints (cytopénies majeures, infections graves répétées), est un résultat thérapeutique important.

Le mécanisme d'action de l'interféron alpha reste totalement inconnu; compte tenu de la cinétique très lente de la cellule et de la diminution très importante et rapide du volume tumoral (chez les patients hyperleucocytaires), l'hypothèse d'une seule inhibition de la prolifération cellulaire reste peu plausible. Une action cytolytique et/ou entrainnant la différenciation des cellules leucémiques a été envisagée. Actuellement, il n'existe pas d'arguments en faveur de l'une ou l'autre de ces hypothèses. Il faut noter que, sous traitement, s'observe chez ces patients une restauration de l'activité NK (natural-killer). La confrontation des études actuellement en cours chez nos patients portant sur le métabolisme intracellulaire de l'interféron, les interactions entre les tricholeucocytes, les cellules lymphoïdes et myéloïdes, l'expression des oncogènes, la cinétique d'apparition d'antigènes de différenciation (classe II d'histocompatibilité), apporteront peut être des réponses à ces questions.

\section{Summary}

Hairy cell leukemia is a rare subset of lymphoid B hemopathy, which is mainly characterized clinically by its frequent and severe infectious complications; these manifestations are related to the degree of granulopenia and monocytopenia.

While splenectomy has improved the prognosis, when splenomegaly was present, chemotherapy has been useless.

Interferon alpha appears to be efficient in correcting myeloid cytopenia and in diminishing tissue infiltration by abnormal cells. Although long term results have yet to be documented, a remarkable success has been observed in severe cases with pancytopenia, and interferon may represent a promising therapy, whatever its origin (purified or recombinant alphainterferon).
G. Flandrin : Hôpital Saint-Louis. 2, place du 40 